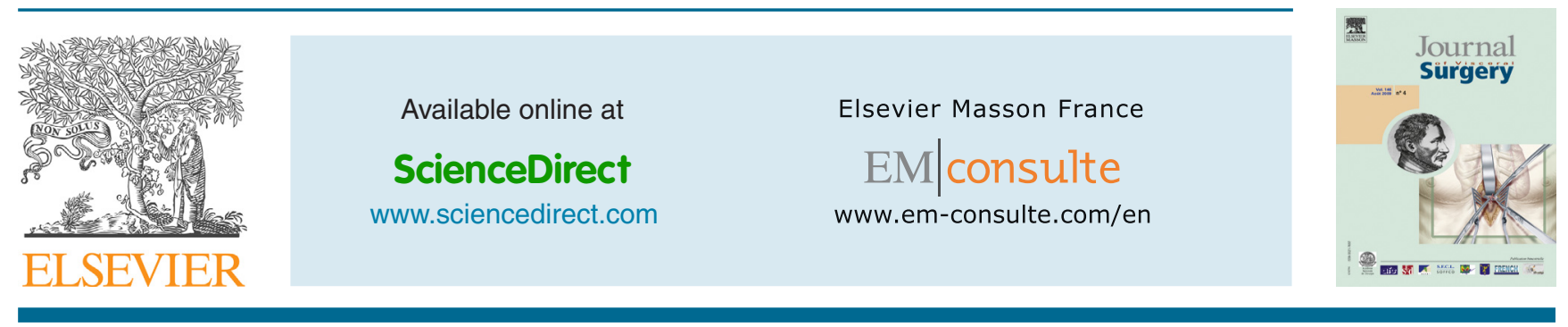

\title{
CORRESPONDENCE
}

\section{For a pre-operative assessment of the colon vascular supply}

\section{Dear Editor,}

We appreciated the paper by Eveno et al. concerning the correlations between extension of the calcifications of the abdominal aorta and the risk of leakage of colorectal anastomoses [1]. We performed a retrospective observational study, based on contrast-enhanced Computed Tomography (CT) imaging, in 30 cases of leakage of left colon anastomoses, 12 left colo-colic and 18 high colo-rectal, clinically relevant (Dindo-Clavien grade III-V) observed in the years 2012-2015 in patients aged between 68 and 80 years. We could identify in 20 cases $(66.66 \%)$ a poor contrast enhancement of colon segments used for the anastomosis, or of the vascular network of the corresponding mesenteries. This was interpreted as a sign of insufficient arterial supply and was considered an important, even if not always the unique, pathogenetic factor of this complication. Therefore, in elective surgery, we deem essential a preoperative study of the arterial anatomy the colon, in order to plan a correct resection and a safe anastomosis. This is particularly important in surgery of the left colon and rectum and can be easily performed by the same imaging CT tool, just used with a general diagnostic aim or for staging a neoplastic disease.

Actually, our policy is to tailor for each case a colon resection, which assures an adequate arterial supply to the anastomosis and is oncologically correct. Some details merit to be underlined.

In case of partial or complete obstruction of the inferior mesenteric artery, of its central ligation upstream the origin of the left colic artery, or of complete mesocolic excision, an adequate arterial collateral network has to be assured by an efficient Drummond's marginal arcade. Similarly, a simple sigmoidectomy with a low tie of the inferior mesenteric artery can be dangerous if the marginal arcade is poorly developed or interrupted: in this case a better blood supply, from the middle colic artery, is obtained if a left hemicolectomy is performed.

In low colo-rectal anastomoses, specific conditions are to be remembered. At first, after a pre-operative radiotherapy, a diffuse fibrosis can involve the mesorectum, with a secondary arteritis jeopardizing the vascular network of the rectum $[2,3]$. Similarly, after a procedure of total mesorectal excision, the blood supply to the residual rectal stump, is provided by the sole inferior haemorrhoidal arteries: in these cases, we prefer a total or sub-total rectal excision, followed by a low colo-rectal or colo-anal anastomosis.

Interestingly, these recommendations, based on principles of vascular anatomy, agree with the general oncological principles.

Clearly, a pre-operative study can be followed by intra-operative controls, with laser Doppler measures or near-infrared light technologies enhanced by fluorescent agents, which can alert about an insufficient, sometimes unexpected, blood supply to the colon or rectum [4].

\section{Disclosure of interest}

The authors declare that they have no competing interest.

\section{References}

[1] Eveno C, Latrasse V, Gayat E, Lo Dico R, Dohan A, Pocard M. Colorectal anastomotic leakage can be predicted by abdominal aortic calcification on preoperative CT scans: a pilot study. J Visc Surg 2016;153:253-7.

[2] Manenti A, Manco G, Vezzelli E, Donatiello S. Vascular risk factors in left colon anastomosis leakage: a computed tomography guided study. WebmedCentral SURGERY 2012;3(5):WMC003346.

[3] Reggiani Bonetti L, Domati F, Farinetti A, Migaldi M, Manenti A. Radiotherapy induced mesorectum alterations: histological evaluations of 90 consecutive cases. Scand J Gastroenterol 2015;50:197-203.

[4] Rutegard M, Rutergard J. Anastomotic leakage in rectal cancer surgery: the role of blood perfusion. World J Gastrointest Surg 2015;7:289-92.

D. Mosca, A. Farinetti , A. Manenti* Department of Surgery, University of Modena, Policlinic Hospital, v.Pozzo, 41121 Modena, Italy

*Corresponding author.

E-mail address: antonio.manenti@unimore.it (A. Manenti) Available online 17 April 2017

http://dx.doi.org/10.1016/j.jviscsurg.2017.02.001 1878-7886/@ 2017 Elsevier Masson SAS. All rights reserved. 